Juan Pablo Quiroga Cuellar Luis Alejandro Lafuente Lafuente ${ }^{2}$

Tarciso Rusivel Rorigues Correia $^{2}$

Gustavo Meireles Salomao ${ }^{2}$ Lizeth Chacon Paco ${ }^{2}$

Correspondencia a:

${ }^{1}$ Cirujano Plástico - Unidad de Quemados del Hospita Niñ@ Manuel Ascencio Villarroel - Complejo Hospitalario Viedma.

Miembro SBCPER - filia Cochabamba - Bolivia

Docente Cátedra Cirugía UNITEPC

${ }^{2}$ Centro de investigación medicina UNITEPC. CIMU Cochabamba - Bolivia

jpqc-med@hotmail.com vicepresidente@socieme. com

presidente@socieme.com gustavomeireles@unitepc-mail.com

lizethchacon@unitepc-mail.com

Procedencia y arbitraje: No comisionado, sometido a arbitraje externo

Recibido para publicación: 1 de Junio del 2018 Aceptado para publicación: 10 de agosto del 2018

Citar como:

$\mathrm{Re}$ Ci Sa UN

2018;5(2):16-22

\section{ACCIDENTES POR QUEMADURAS EN EDAD PEDIÁTRICA}

\section{BREAST ACCIDENTS IN PEDIATRIC AGES}

\section{ACIDENTES DE QUEIMADURAS EM IDADE PEDIÁTRICA}

\section{Resumen.}

Los accidentes por quemaduras en la edad pediátrica son un problema de salud pública de alta morbimortalidad, si no son manejados adecuadamente pueden dejar secuelas invalidantes, el objetivo del estudio fue, identificar la incidencia de los accidentes por quemaduras según el sexo, edad, causa y la gravedad.

Se revisó 332 historias clínicas de pacientes ingresados al servicio especializado de quemados del Hospital del Niño Manuel Ascencio Villarroel del complejo Hospitalario VIEDMA, en el departamento de Cochabamba / Bolivia, en el año 2017.

La incidencia es de 175 niños (53\%) y 157 niñas (47\%). Según los grupos etarios: de 1 a 5 años 215 (65\%), 5 a 10 años 69 (21\%), menores de un año $29(9 \%)$ y mayores de 10 años 19 (5\%). Las causas fueron: líquidos calientes 215 (65\%), contacto directo con fuego 62 (19\%), objetos calientes 27 (8\%), electricidad $20(6 \%)$, fricción $7(2 \%)$ y 1 por quemadura por químicos. La gravedad presento los siguientes resultados; el grado A: leve $8(2 \%)$, Moderado 0, Grave 0, Critico $1(0 \%)$. El AB: leve 109 (33\%), Moderado 125 (38\%), Grave 60 (18\%), Critico 9 (3\%) y el B: leve 4 (1\%), Moderado 13 (4\%), Grave $3(1 \%)$, Critico 0. Estos datos se relacionan con otros trabajos que se realizaron en otros países.

Palabras clave: Incidencia, Quemaduras, Niños

\section{Summary.}

Accidents due to burns in the pediatric age are a public health problem with high morbidity and mortality. If they are not managed properly, they can leave invalidating sequels. The objective of the study was to identify the incidence of burns accidents according to sex, age, cause and the gravity.

We reviewed 332 medical records of patients admitted to the specialized burn service of the Manuel Ascencio Villarroel Children's Hospital of the VIEDMA Hospital Complex, in the department of Cochabamba / Bolivia, in the year 2017.

The incidence is 175 children (53\%) and 157 girls (47\%). According to the age groups: from 1 to 5 years old 215 (65\%), 5 to 10 years old $69(21 \%)$, younger than one year $29(9 \%)$ and older than 10 years old $19(5 \%)$. The causes were: hot liquids $215(65 \%)$, direct contact with fire 62 (19\%), hot objects 27 (8\%), electricity $20(6 \%)$, friction $7(2 \%)$ and 1 for chemical burn. The gravity presented the following results; grade A: mild 8 (2\%), Moderate 0 , 
Severe 0, Critical 1 (0\%). The AB: mild 109 (33\%), Moderate 125 (38\%), Severe 60 $(18 \%)$, Critical $9(3 \%)$ and B: mild $4(1 \%)$, Moderate $13(4 \%)$, Serious $3(1 \%)$, Critical 0 . These data are related to other works that were carried out in other countries.

Keywords: Incidence, Burns, Children

\section{Resumo.}

Os acidentes por queimaduras em crianças é um problema de saúde pública de alta morbidade e mortalidade, se não for devidamente controlado pode deixar seqüelas incapacitantes. O objetivo do estudo foi identificar a incidência de acidentes de queimaduras por sexo, idade, causa e gravidade.

Foi revisado 332 histórias clinicas de pacientes internados ao serviço especializado em queimados do Hospital Infantil Manuel Ascencio Villarroel do Complexo Hospitalario VIEDMA, no departamento de Cochabamba / Bolívia, em 2017.

A incidência é de 175 meninos (53\%) e 157 meninas (47\%). De acordo com o grupo de idade de 1 a 5 anos 215 (65\%), 5 a 10 anos 69 (21\%), inferior a um ano 29 (9\%) e com idades entre 10 anos $19(5 \%)$. As causas foram: líquidos quentes 215 (65\%), contacto directo com o fogo $62(19 \%)$, objetos quentes $27(8 \%)$, de eletricidade 20 $(6 \%)$, fricçao 7 (2\%) e uma queimadura química. A gravidade apresentou os seguintes resultados: para tipo $A$ : leve $8(2 \%)$, moderada 0 , severa 0 , crítica $1(0 \%)$. AB: leve $109(33 \%)$, Moderada $125(38 \%), 60$ grave (18\%), critica $9(3 \%)$ e B: leve $4(1 \%)$, Moderada 13 (4\%), 3 grave (1\%), crítica 0 . Esses dados estão relacionados a outros trabalhos que foram realizados em outros países.

Palavras-chave: Incidência, Burns Children

\section{INTRODUCCIÓN}

Las quemaduras son lesiones en la piel causadas por agentes físicos, biológicos, químicos y radioactivos, que al contacto con la piel pueden ocasionar daño en menor o mayor grado. La severidad de una quemadura esta en relación a la profundidad, área en superficie y la localización de la lesión.

Es uno de los mayores traumas que las personas pueden sufrir, estos son una de las principales causas de accidentes en niños. La gran mayoría prevenibles, ya que son producto de descuidos o no conocen los riesgos en algunas situaciones. Por esta razón los niños son los más vulnerables y deben recibir más atención especializada en su cuidado. ${ }^{1}$

También estas quemaduras en niños crean un gran problema, ya que estos presentan un alto riesgo de morbimortalidad, dejando lesiones invalidantes, funcionales y estéticas. $^{2}$

También significan un alto costo para el estado, debido a que estas lesiones requieren periodos largos de hospitalización y atención multidisciplinaria. ${ }^{3}$

La quemadura deja de ser una alteración local cuando esta es mayor a ciertos parámetros, convirtiéndose así en la enfermedad por quemadura. La recuperación de un paciente quemado depende de la fuente de la quemadura, intensidad, edad del paciente, enfermedades previas, tiempo y calidad de atención en la etapa aguda. ${ }^{4}$ 
Según la profundidad y capa de piel lesionada es clasificada por el Dr. Benaím en tres tipos de quemaduras..$^{5,6}$

1.- Quemaduras de tipo A o superficial: (primer grado, epidérmica), afecta solamente la epidermis. Se distinguen dos formas:

- Tipo A eritematosa: se presenta como un enrojecimiento de la piel (eritema) sin ruptura de la misma; presenta hipersensibilidad. El ejemplo más frecuente es la exposición al sol.

- Tipo A flictenular: está involucra a la membrana basal, sin alterar la dermis. La característica es la flictena, presenta hipersensibilidad.

2.- Quemaduras de tipo AB o intermedia: (segundo grado, dérmica) involucra a la dermis. En las quemaduras intermedias, según sea lesionada la dermis se puede estimar la evolución. Se distinguen dos formas:

- Tipo AB-A (quemaduras intermedio-superficiales) en esta la piel se regenera a partir de los restos epidérmicos de las faneras.

- Tipo AB-B (quemaduras intermedio-profundas) por la mayor destrucción de la dermis requieren injerto de piel para su curación.

3.- Quemaduras de tipo B (tercer grado, subdérmica), la lesión se extiende hasta la hipodermis, todas las capas de la piel son lesionadas. Es necesario injerto y/o colgajo para la reparación de la lesión.

Para muchos autores las quemaduras de cuarto grado son aquellas que afectan el tejido subcutáneo, musculo, fascia, periostio o hueso. Pero por lo general no se utiliza esta última denominación. ${ }^{5,6}$

La Superficie Corporal Quemada Total (SCQT) es determinada por diferentes tipos de métodos: $:^{7,8}$

- Regla de la palma de la mano: La cara palmar de la mano del paciente representa el $1 \%$ de la superficie corporal. Recordemos que esta regla se refiere al total de la cara palmar incluidos los dedos, sirve para quemaduras de superficie y distribución irregular.

- Regla de los nueve o Esquema de Lund Browder: esta se utiliza en adultos, a los distintos segmentos corporales les da un valor de $9 \%$ y $1 \%$ genitales.

La gravedad de la quemadura se determina: según el porcentaje de SCQT y tipo de quemadura, para esto se utiliza la tabla de Benaím, esta establece cuatro grupos. ${ }^{7,8}$

\begin{tabular}{|c|c|c|c|c|}
\hline $\begin{array}{l}\text { Grupo de gravedad } \\
\text { según porcentaje de } \\
\text { superficie corporal }\end{array}$ & $\begin{array}{l}\text { Grupo I } \\
\text { Leve }\end{array}$ & $\begin{array}{c}\text { Grupo II } \\
\text { Moderado }\end{array}$ & $\begin{array}{c}\text { Grupo III } \\
\text { Grave }\end{array}$ & $\begin{array}{c}\text { Grupo IV } \\
\text { Critico }\end{array}$ \\
\hline $\begin{array}{l}\text { A (1 }{ }^{\text {er }} \text { Grado, Super- } \\
\text { ficial) }\end{array}$ & Hasta $15 \%$ & 16 al $30 \%$ & 31 al $60 \%$ & Mas del $60 \%$ \\
\hline $\begin{array}{l}A B\left(2^{\text {do }} \text { Grado, Inter- }\right. \\
\text { media) }\end{array}$ & Hasta $5 \%$ & 6 al $15 \%$ & 16 al $45 \%$ & Mas del $45 \%$ \\
\hline $\begin{array}{l}\text { B (3 } 3^{\text {er }} \text { Grado, Profun- } \\
\text { da) }\end{array}$ & Hasta $1 \%$ & 2 al $5 \%$ & 6 al $20 \%$ & Mas del $20 \%$ \\
\hline
\end{tabular}

La curación de la lesión depende de la profundidad y la extensión, aunque hay zonas que, por su ubicación como manos, pies, cara y perineo, producen limitaciones e incapacidades importantes. ${ }^{7,8}$ 
El objetivo del presente estudio es identificar la incidencia de los accidentes por quemaduras según el sexo, edad, causa, y la gravedad de la quemadura en niños que acudieron al servicio especializado de quemados del Hospital del Niño Manuel Ascencio Villarroel del complejo Hospitalario VIEDMA, en el departamento de Cochabamba / Bolivia.

\section{METODOLOGÍA}

Se realizó un estudio de tipo cuantitativo, retrospectivo, de corte transversal. Se revisó las historias clínicas de los pacientes ingresados en año 2017 al servicio especializado de quemados del Hospital del Niño Manuel Ascencio Villarroel del complejo Hospitalario VIEDMA, en el departamento de Cochabamba / Bolivia.

La muestra estuvo conformada por 332 historias clínicas, que cumplieron con todos los criterios de inclusión.

El área de estudio fue en niños y niñas menores de 14 años con quemaduras que fueron ingresados al servicio especializado de quemados.

La técnica de recolección de datos, fue realizada mediante la revisión de historias clínicas de los pacientes y llenado de base de datos en el programa IBM SPSS.

\section{RESULTADOS}

Se tomaron 332 historias clínicas comprendidas entre los meses enero a diciembre del año 2017, de pacientes que fueron ingresados al servicio especializado de quemados del Hospital del Niño Manuel Ascencio Villarroel del complejo Hospitalario VIEDMA, en el departamento de Cochabamba, y los resultados mostraron lo siguiente:

La incidencia de niños con accidentes por quemaduras es de 175 niños (53\%), y 157 niñas (47\%). Ver cuadro 1.

\section{Cuadro 1.}

Incidencia de accidentes por quemaduras según el sexo.

\begin{tabular}{lcc}
\hline Sexo & Incidencia & $\%$ \\
\hline Femenino & 157 & $47 \%$ \\
Masculino & 175 & $53 \%$ \\
\hline
\end{tabular}

Según los grupos etarios los que más accidentes por quemaduras presentaron fue, el grupo de 1 a 5 años con 215 casos (65\%), y le sigue el de 5 a 10 años con 69 casos (21\%), el grupo de menores de un año es de 29 casos (9\%) y por último el grupo de mayores de 10 años con 19 casos (5\%). Ver cuadro 2.

\section{Cuadro 2.}

Incidencia de accidentes por quemaduras según grupos etarios.

\begin{tabular}{lcc}
\hline Edad & Incidencia & $\%$ \\
\hline$<1$ año & 29 & 9 \\
1 a 5 años & 215 & 65 \\
5 a 10 años & 69 & 21 \\
$>10$ años & 19 & 5 \\
\hline
\end{tabular}

Las causas de los accidentes por quemaduras mostraron que la principal causa es por líquidos calientes 215 casos (65\%), y le siguen contacto directo con fuego 62 
casos (19\%), objetos calientes 27 casos (8\%), electricidad 20 casos (6\%), fricción 7 casos $(2 \%)$ y solo 1 caso por quemadura por químicos. Ver cuadro 3.

Cuadro 3.

Causas de los accidentes por quemaduras.

\begin{tabular}{lcc}
\hline Causa & Incidencia & $\%$ \\
\hline Liquido Caliente & 215 & 65 \\
Fuego & 62 & 19 \\
Objeto Caliente & 27 & 8 \\
Electricidad & 20 & 6 \\
Fricción & 7 & 2 \\
Químicos & 1 & 0 \\
\hline
\end{tabular}

La gravedad de la quemadura se determina según el porcentaje de Superficie Corporal Quemada Total SCQT y tipo de quemadura. Para esto se utiliza la tabla de Benaím, teniendo los siguientes resultados: el grado A presento: Leve 8 casos (2\%), Moderado 0 casos, Grave 0 casos, Critico 1 caso (0\%). El AB presento: Leve 109 casos (33\%), Moderado 125 casos (38\%), Grave 60 casos (18\%), Critico 9 casos (3\%) y el B: Leve 4 casos (1\%), Moderado 13 casos (4\%), Grave 3 casos (1\%), Critico 0 casos. Ver cuadro 4.

Cuadro 4.

Gravedad de los accidentes por quemaduras

\begin{tabular}{|c|c|c|c|c|}
\hline $\begin{array}{l}\text { Grupo de gravedad } \\
\text { según porcentaje de } \\
\text { superficie corporal }\end{array}$ & $\begin{array}{l}\text { Grupo I } \\
\text { Leve }\end{array}$ & $\begin{array}{l}\text { Grupo II } \\
\text { Moderado }\end{array}$ & $\begin{array}{c}\text { Grupo III } \\
\text { Grave }\end{array}$ & $\begin{array}{c}\text { Grupo IV } \\
\text { Critico }\end{array}$ \\
\hline $\begin{array}{l}\text { A (1 } 1^{e r} \text { Grado, Superfi- } \\
\text { cial) }\end{array}$ & $8(2 \%)$ & 0 & 0 & 1 \\
\hline $\begin{array}{l}A B\left(2^{d o} \text { Grado, Interme- }\right. \\
\text { dia) }\end{array}$ & $109(33 \%)$ & $125(38 \%)$ & $60(18 \%)$ & $9(3 \%)$ \\
\hline B (3er Grado, Profunda) & $4(1 \%)$ & $13(4 \%)$ & $3(1 \%)$ & 0 \\
\hline
\end{tabular}

\section{DISCUSIÓN}

Según Frías y Artiga, la tasa de morbilidad por quemaduras ha disminuido en la última década, lo cual está relacionado con la aplicación de los programas encaminados tanto a la prevención del accidente como a la promoción de salud en el nivel primario de atención, así como a la permanente educación sanitaria de los profesionales que durante muchos años han capacitado a padres, familiares y a la población en general acerca de este tema. Los niños tienen que tener un cuidado especifico, por ser más frecuente a sufrir estos accidentes; en Bolivia, los accidentes domésticos aún constituyen un importante problema de salud pública. ${ }^{9,10 .}$

Este estudio mostró que las lesiones son mucho más frecuentes en los niños $53 \%$ que en las niñas 47\%, y las explicaciones que ofrece según Borges, para ello son diversas, por ejemplo: el carácter más travieso del varón que puede constituir un factor 
predisponente a los accidentes. ${ }^{11 .}$

El grupo etario que más accidentes por quemaduras presento fue, el grupo de 1 a 5 años con un $65 \%$, en comparación a la literatura que reporta que los niños de 1 a 4 años son el grupo de más alto riesgo. ${ }^{12}$.

Entre las causas más frecuentes por quemadura fue el de líquidos calientes $65 \%$, y le siguen contacto directo con fuego $19 \%$, objetos calientes $8 \%$, electricidad $6 \%$ y fricción $2 \%$. Estas causas se relacionan con los resultados de una publicación realizada sobre la epidemiología de las quemaduras en la infancia en el cual se encontró que las producidas por líquidos representaron 48,2 \%, seguidas por las ocasionadas por el fuego $32,7 \%$ y con otros estudios realizados sobre el tema. ${ }^{13,14,15}$.

En relación a la gravedad de la quemadura tiene mayor frecuencia el grado $A B$ en sus diferentes niveles: Leve 33\%, Moderado 38\%, Grave 18\%, Critico 3\%, este resultado se relaciona con las publicaciones de Abad y Garzon, que indican lo mismo. ${ }^{16,17}$

Los accidentes que llevan a las quemaduras son prevenibles y de una elevada morbimortalidad, que conllevan altos costos en salud y discapacidad permanente en los niños. El grupo que realizo este trabajo estableció que se realicen dos ferias anuales de prevención de las quemaduras en niños, que estarán a cargo del servicio especializado de quemados del Hospital del Niño Manuel Ascencio Villarroel del complejo Hospitalario VIEDMA y la Sociedad Científica de Estudiantes de Medicina de la Universidad Técnica Privada Cosmos UNITEPC.

\section{BIBLIOGRAFÍA}

1. Meda Vendrusculo T, Baldin Balieiro CR, Echevarría Guanilo ME, Farina Junior JA, Aparecida RossiL. Quemaduras en ambiente doméstico: características y circunstancias del accidente. Rev Latino Am Enfermagem. Jun 2010;18(3)160-68.

2. Sánchez López JE. Manejo del niño quemado. Rev Cient Cienc Méd. 2011;14(2):2830.

3. Koc Z, Saglam Z. Burn epidemiology and cost of medication in pediatric burn patients. Buns. 2012 Sep;38(6):813-9.

4. Orozco Valerio MJ, Miranda Altamirano RA, Méndez Magaña AC, Celis A. Tendencia de mortalidad por quemaduras en México.1979-2009. Gaceta Médica de México. 2012; 148:349-57.

5. Linares HA, Benain F. (1993). Tratado de quemaduras. Buenos Aires: Ed. Interamericana, p.368-371.

6. Benaim F. (2004). Consideraciones sobre la evaluación de la gravedad de las quemaduras. RAQ, Ab, 18: 26-30.

7. Abad P, Acosta D, Martínez I, Lloret J, Patiño B, Gubert L, Carol J, Boinx O. (2000). Quemaduras. Cir.

8. Garcés S, Artigas R. (1995). Quemaduras. Sociedad de Cirujanos de Chile. ISBN 956-7450-02-7. http://tesis.luz.edu.ve/tde_arquivos/16/TDE-2011-1101T09:06:53Z 2068/Publico/urdaneta_lima_eleazar.pdf

9. Frías Méndez E. Quemaduras. Diagnóstico y tratamiento en las primeras 72 horas. Rev. Cubana Cir. 2006 [citado 12 Dic 2013];45(2).

10. Artigas Nambrard R. Tratamiento de las quemaduras en los niños. Rev. Chilena 
Pediatría. 1953;24(7):235-42.

11. Borges Muñio $H$. Manual de procedimientos diagnóstico y tratamiento en traumatología y Cirugía Plástica. La Habana: Editorial Pueblo y Educacion; 1984.p. 7-40.

12. Hu GQ, Zhu SL, Wang QQ, et al: An epidemiological survey on the incidence of non fatal injury and influencing factors among children under 5 years old in China. Zhonghua Liu Xing Bing Xue Za Zhi 2011; 32 (8): 773-6.

13. Martínez L, Ros Z, López JC, Díaz M, Quezada B, Perdiguero M, et al. La dermis artificial (íntegra) en Cirugía reconstructiva pediátrica. Cir Pediatr. 2002 [citado 12 Dic 2013]; 15:97-100.

14. De los Santos González CE. Guía básica para el tratamiento del paciente quemado [citado 12 Dic 2013].

15. Theodorou P, Maurer CA, Spanholtz TA. Acalculous cholecystitis in severely burned patients: incidence and predisposing factors. Burns. 2009; 35:405-11.

16. P. Abad, D. Acosta, V. Martínez-Ibáñez, J. Lloret, B. Patiño, Gubern LI, et al. Quemaduras en la infancia. Trascendencia social a las puertas del 2000 Cir Pediatr., 13 (2000), pp. 97-101 View Record in Scopus

17. E. Garzón-Guerrero, M. Repizo-Correa, A. Sabogal-Amado, N. Suárez-Acosta, D. Lozano-Poveda Características epidemiológicas de niños de 0 a 15 años con quemaduras Investigación en Enfermería: Imagen y Desarrollo., 1 (1999), pp. 61-64 View Record in Scopus 